\title{
Initiative für Pflegekammer
}

\author{
Mit möglichst vielen Unterschriften zur politischen Kraft
}

Als gemeinsame Plattform Pflegebezogener Interessensvertretungen und Berufsgruppen setzt sich die Österreichische Pflegekonferenz für ein effektives und effizientes Gesundheitssystem im Interesse der Bevölkerung und der Pflegepersonen ein. Mitglieder der Österreichischen Pflegekonferenz (ÖPK) sind derzeit die Arbeitsgemeinschaft hämato-onkologischer Pflegepersonen in Österreich (AHOP), der Berufsverband Kinderkrankenpflege (BKKÖ), der Berufsverband österreichischer Gesundheits- und Krankenpflegeberufe (BoeGK), der Österreichische Berufsverband für Anästhesie- und Intensivpflege (ÖBAI), der Österreichische Gesundheits- und Krankenpflegeverband (ÖGKV) und die Österreichische Gesellschaft für vaskuläre Pflege.

Im vergangenen Dezember vereinbarten die Mitglieder der ÖGP, die Bürgerinitiative des BoeGK zur Gründung einer Pflegekammer gemeinsam und vollinhaltlich zu unterstützen, berichtet Franz Allmer, der Präsident des BoeGK. Die Unterstützungserklärung wurde nun entsprechend der Vereinbarung angepasst und kann von den Internetseiten der Mitgliederorganisationen ebenso wie von jener der ÖPK heruntergeladen und vervielfältigt werden. Die Unterstützung durch die ÖPK bietet der Bürgerinitiative zur Gründung einer Pflegekammer in Österreich nun eine wesentlich breitere Basis. Gemeinsam sollen Konzepte und weitere Vorgehensweisen entwickelt und vereinbart werden.

\section{Bedarf an qualifizierter Pflege steigt}

In Österreich, so Allmer, gibt es derzeit 662.000 Menschen, die älter als 75 Jahre sind, die Zahl wird 2030 die Millionengrenze übersteigen. Betreut werden sie bei Pflegebedürftigkeit in der überwiegenden Mehrheit von nahen Angehörigen - die jedoch häufig überfordert sind. Je besser die Beratung und professionelle Unterstützung im extramuralen Bereich funktioniert, desto länger ist es möglich, den Verbleib dieser Personen daheim im gewohnten Umfeld zu erhalten. Um der hochkomplexen Problematik der zukünftigen Betreu-

ung und Pflege chronisch Kranker, hochbetagter, schwerkranker und behinderter Menschen aller Altersgruppen zu begegnen, stellt Allmer fest, benötigen wir weitblickende Konzepte unter Einbeziehung der Bedürfnisse der betroffenen Menschen und deren Angehörigen: „Dazu müssen die Pflegeberufe zukünftig eine politische Struktur bekommen, um in die Gesundheitsplanung der Zukunft, im intra- wie extramuralen Bereich, verpflichtend einge-

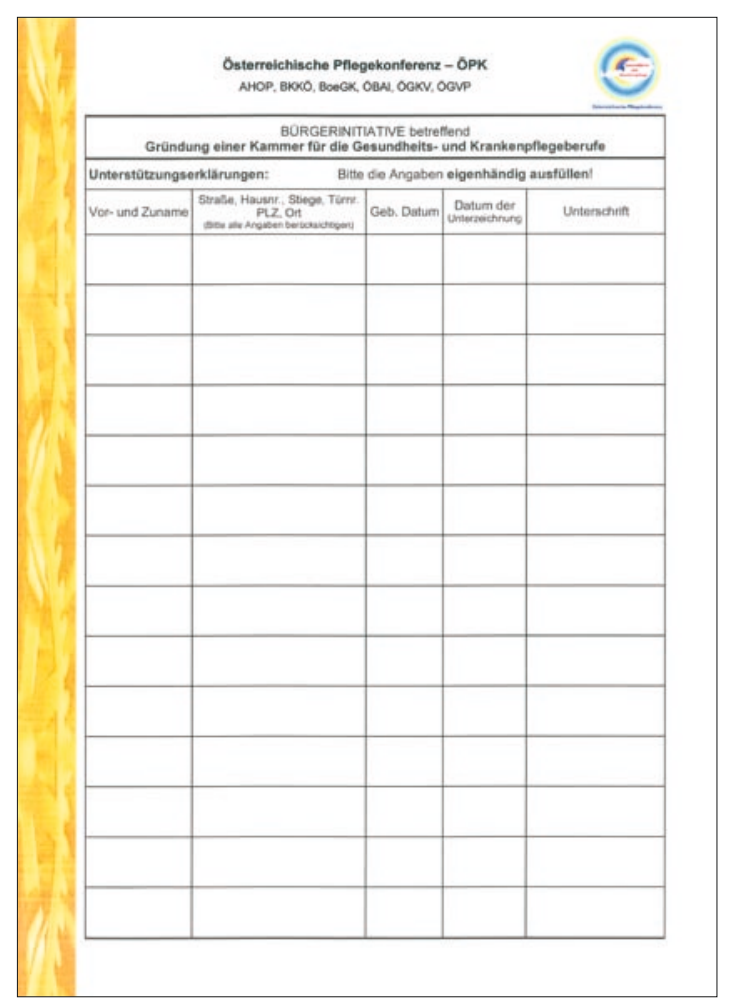

Damit nicht mehr Berufsfremde über die Pflege entscheiden: Bürgerinitiatvie für eine Pflegekammer.

bunden zu werden."

Berufsverbände als Organisationen mit freiwilliger Mitgliedschaft werden von Politikern aufgrund ihrer vermeintlich nicht gegebenen Vertretungsbreite nicht ausreichend eingebunden. Das Ergebnis: Berufsfremde entscheiden in der Mehrzahl der Fälle über die Pflegeberufe. Damit, so Allmer, ist jedoch die Entwicklung des Berufes und der notwendigen Veränderungsmaßnahmen nicht gegeben: „Die Arbeitnehmerinteressen werden von der Arbeiterkammer und der Gewerkschaft zu 100
Prozent abgedeckt, die beruflichen Interessen jedoch nur unzureichend wahrgenommen." Grund genug, für die einzelnen Berufsverbände diese Situation verändern zu wollen und durch die Bündelung der Kräfte in der Österreichischen Pflegekonferenz eine politische Struktur in Form einer Pflegekammer zu fordern. Sie soll die Ziele und Forderungen der Gesundheits- und Krankenpflegeberufe auf eine rechtlich verbindliche Basis stellen und so die Einbeziehung der Gesundheits- und Krankenpflegeexperten in die strategische Entwicklung der Gesundheitspolitik für die Zukunft sichern. Den Angehörigen der Gesundheitsund Krankenpflegeberufe würden als Mitglieder der Pflegekammer keine zusätzlichen Aufwendungen entstehen, da diese Mitgliedschaft jene bisher bestehende bei der Arbeiterkammer ersetzt.

Unterstützungsliste aus dem Internet holen

Die Bürgerinitiative unterstützen kann jeder österreichische Staatsbürger der das 16. Lebensjahr vollendet hat mit seiner Unterschrift. Entsprechende Unterschriftenlisten sind über die Internetseiten der in der Österreichischen Pflegekonferenz vertretenen Pflegeorganisationen auszudrucken: www.boegk.at, www. oegkv.at, www.ahop.at, www. kinderkrankenpflege.at, www. oebai.at, www.pflegekonferenz.at, www.oegvp.at. Bis 14. März müssen die Unterschriftenlisten beim Initiator, dem Berufsverband der österreichischen Gesundheits- und Krankenpflege, 1095, Postfach 67 eingelangt sein.

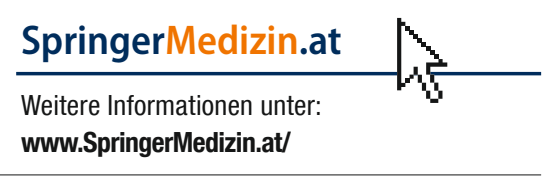

